\title{
Comparison of discrete event simulation tools in an academic environment
}

\author{
Mario Jadrić ${ }^{1, *}$, Maja Ćukušić ${ }^{1}$ and Antonia Bralić ${ }^{1}$ \\ ${ }^{1}$ Faculty of Economics, University of Split \\ Cvite Fiskovića 5, 21000 Split, Croatia \\ E-mail: 〈\{mario.jadric, maja.cukusic, antonia.bralic\}@efst.hr〉
}

\begin{abstract}
A new research model for simulation software evaluation is proposed consisting of three main categories of criteria: modeling and simulation capabilities of the explored tools, and tools' input/output analysis possibilities, all with respective subcriteria. Using the presented model, two discrete event simulation tools are evaluated in detail using the task-centred scenario. Both tools (Arena and ExtendSim) were used for teaching discrete event simulation in preceding academic years. With the aim to inspect their effectiveness and to help us determine which tool is more suitable for students i.e. academic purposes, we used a simple simulation model of entities competing for limited resources. The main goal was to measure subjective (primarily attitude) and objective indicators while using the tools when the same simulation scenario is given. The subjects were first year students of Master studies in Information Management at the Faculty of Economics in Split taking a course in Business Process Simulations (BPS). In a controlled environment - in a computer lab, two groups of students were given detailed, step-by-step instructions for building models using both tools - first using ExtendSim then Arena or vice versa. Subjective indicators (students' attitudes) were collected using an online survey completed immediately upon building each model. Subjective indicators primarily include students' personal estimations of Arena and ExtendSim capabilities/features for model building, model simulation and result analysis. Objective indicators were measured using specialised software that logs information on user's behavior while performing a particular task on their computer such as distance crossed by mouse during model building, the number of mouse clicks, usage of the mouse wheel and speed achieved. The results indicate that ExtendSim is well preferred comparing to Arena with regards to subjective indicators while the objective indicators are better for Arena. Objectively, students completed the given scenario faster and with fewer movements in Arena, but they still prefer ExtendSim and perceive it as a better tool considering the characteristics and functionalities.
\end{abstract}

Key words: discrete event simulation, tool evaluation, ExtendSim, Arena

Received: September 16, 2014; accepted: December 2, 2014; available online: December 30, 2014

${ }^{*}$ Corresponding author. 


\section{Introduction}

Computer simulation is an extremely useful tool for evaluating business processes, as well as for developing and comparing different strategies and observing their outcomes. Numerous authors have confirmed that computer simulation is a valuable and appreciated tool in decision-making processes. For example, Pidd [10] writes: "computer simulation is one of the success stories of operational research" and Hlupić [5] states that there indeed could exist different strategies for achieving performance improvement and operating costs reduction, and investigation of these strategies is well facilitated by simulation. A similar remark was made by Bosilj-Vukšić et al. [2], who investigate business process modeling and related evaluations and emphasize that a method chosen for business process modeling "should enable process evaluation and selection of alternatives" and in regard to that, simulation of discrete events should be considered as an appropriate method. In a similar note, Gupta et al. [4] state that simulation is a powerful tool that enables business process designers to visualize a new system and to quantify and observe behaviors of real systems.

When considering different types of computer simulation, it is important to differ between discrete and continuous simulations, as two main types of dynamic computer simulations. Both types of computer simulation enable researchers or analysts to observe the model, its behavior and state, as well as to evaluate the output during the complete duration of the experiment (including moments of stability as well as moments of transition). A significant impact of teaching and using simulations in academic environments has been well noticed and documented, more and more with evolution of technology enhanced learning environments and tools. By using simulations, students are empowered and taught to make decisions throughout the learning process regardless of the topic at hand. Generally, simulations are more interesting and engaging than traditional learning methods and therefore lower course drop-out rates are noted. Similar to corporate surrounding, where Hlupić [5] states that simulation can be used in different areas of work, which is the reason why there is a rather large number of simulation packages available on the software market today, an academic environment also deals with numerous simulation software solutions that could be implemented and used for educational purposes. Therefore, a need to evaluate the effectiveness and outcomes of using different simulation tools comes up for educators, especially in the higher education area.

Tools that are investigated in this paper are Arena® Simulation Software by Rockwell Automation (USA) and ExtendSim by Imagine That (USA). Both are widely used tools for discrete event simulation, and have been used for teaching discrete event simulation by the authors of the paper in an academic 
environment. We hypothesized that students perform differently using different tools even if they model the same process. Students' modeling experience, simulation and output analysis performance and satisfaction were collected, analyzed and compared in order to choose a more suitable simulation tool for the next generations of students.

\section{The model of simulation software evaluation}

The authors bring forth different categories and ways of evaluating simulation software, some of which inspect the possibility of using simulations as a feature in a business process modeling tool. This context of use is of particular importance for the research at hand since the simulation tools in the presented setting are used solely for purposes of business process simulations. Two models were closely assessed to find out which one is most appropriate as the theoretical basis for research. Bradley et al. [3] (in [9]) listed several categories in order to "evaluate business process re-engineering software tools": (i) Tool capabilities, (ii) Tool hardware and software, (iii) Tool documentation, (iv) User features, (v) Modeling capabilities, (vi) Simulation capabilities, (vii) Output analysis and BPR expertise. Bosilj-Vukšić et al. [2] formed an evaluation model consisting of four main groups of categories and several sub-categories: (i) Hardware and software considerations (encompassing: coding aspects, software compatibility, user support, financial and technical features, pedigree), (ii) Modeling capabilities (with: general features, modeling assistance), (iii) Simulation capabilities (through: visual aspects, efficiency, testability, experimentation facilities, statistical facilities), and (iv) Input/output issues (listing: input and output capabilities, analysis capabilities). Verma and Gupta [11] use the same four categories to evaluate software tools in their research. For purposes of this research, the two models were combined and adapted in order to compare simulation tools with respect to their similarity. Here we provide an overview of the chosen models and explain how they are combined to fit in the new research model. Bosilj-Vukšić et al. [2] based their model on a framework developed by Hlupić et al. [6] and adapted it to the domain of business process modeling software. The adaptation is important for the context of our research in regard to the modeled process's characteristics. On the other hand, JansenVullers \& Netjes [9] strived to evaluate BPS software packages elaborating on the categories defined by Bradley et al. [3], with particular interest on three of the categories, which are in fact common for the original and the derived model. In Table 1, these models are described and adapted categories are presented. Categories in the second model are re-arranged solely to fit better to the presentation of the new model. 


\begin{tabular}{|c|c|c|}
\hline $\begin{array}{l}\text { Model } 1 \\
\text { Presented in [2] }\end{array}$ & $\begin{array}{l}\text { Model } 2 \\
\text { Presented in [3], [9] }\end{array}$ & Proposed research model \\
\hline $\begin{array}{l}\text { A1. Hardware and } \\
\text { software } \\
\text { considerations } \\
\text { A1.1. Coding aspects } \\
\text { A1.2. Software } \\
\text { compatibility } \\
\text { A1.3. User support } \\
\text { A1.4. Financial and } \\
\text { technical features } \\
\text { A1.5. Pedigree }\end{array}$ & $\begin{array}{l}\text { B1. Tool capabilities } \\
\text { - a rough indication of modelling, } \\
\text { simulation and analysis } \\
\text { capabilities }\end{array}$ & $\begin{array}{l}\text { General information } \\
\text { - students' subjective estimation of } \\
\text { their own theoretical and practical } \\
\text { knowledge of BPS }\end{array}$ \\
\hline $\begin{array}{l}\text { A2. Modelling } \\
\text { capabilities } \\
\text { A2.1. General features } \\
\text { A2.2. Modelling } \\
\text { assistance }\end{array}$ & $\begin{array}{l}\text { B2. Modelling capabilities } \\
\text { B2.1. Identification of different roles } \\
\text { B2.2. Model integrity analysis } \\
\text { B2.3. Model flexibility } \\
\text { B2.4. Level of detail }\end{array}$ & $\begin{array}{l}\text { Modelling capabilities } \\
\text { Modeling process } \\
\text { M1. Using the tool - A2.1., B7.1. } \\
\text { M2. Procedure of model building } \\
\text { M3. Studying how to use the tool - } \\
\text { A2.1. } \\
\text { M4. Model verification - B2.2. } \\
\text { M5. Importing resources into a model } \\
\text { M6. Importing data into a model } \\
\text { M7. Adding and editing model data } \\
\text { Modeling support } \\
\text { M8. Number and types of templates } \\
\text { A1.3. } \\
\text { M9. Level of detail during modeling } \\
\text { A3.2., B2.4. } \\
\text { M10. Intuitiveness of objects } \\
\text { M11. Automatic tracking of basic } \\
\text { model performance } \\
\text { M12. Automatic tracking of a larger } \\
\text { number of experiments } \\
\text { M13. On-line help - A2.2., B6.2. }\end{array}$ \\
\hline $\begin{array}{l}\text { A3. Simulation } \\
\text { capabilities } \\
\text { A3.1. Visual aspects } \\
\text { A3.2. Efficiency } \\
\text { A3.3. Testability } \\
\text { of the workflow path } \\
\text { A3.4. Experimentation } \\
\text { facilities } \\
\text { A3.5. Statistical } \\
\text { facilities }\end{array}$ & $\begin{array}{l}\text { B3. Simulation capabilities, } \\
\text { B3.1. Summarizing the nature of } \\
\text { simulation } \\
\text { B3.2. Handling of time and cost } \\
\text { aspects } \\
\text { B3.4.Handling of statistical } \\
\text { distributions }\end{array}$ & $\begin{array}{l}\text { Simulation capabilities } \\
\text { S1. Performance display (resource/time } \\
\text { usage, quality) - B3.2. } \\
\text { S2. Importing and choosing probability } \\
\text { distribution - B3.4. } \\
\text { S3. Model animation - A3.1., A3.1. } \\
\text { S4. Adaptability to model changes } \\
\text { S5. Reliability (animation) - A3.2. } \\
\text { S6. Speed of executing the simulation - } \\
\text { A3.4. } \\
\text { S7. Dynamic objects of the model - } \\
\text { A3.3. } \\
\text { S8. Dynamic display of statistical data }\end{array}$ \\
\hline $\begin{array}{l}\text { A4. Input/Output } \\
\text { issues } \\
\text { A4.1. Input and } \\
\text { output capabilities } \\
\text { A4.2. Analysis } \\
\text { capabilities }\end{array}$ & $\begin{array}{l}\text { B4. Output analysis capabilities } \\
\text { B4.1. Output analysis } \\
\text { B4.2. BPR expertise }\end{array}$ & $\begin{array}{l}\text { Capabilities of output analysis } \\
\text { O1. Report's format } \\
\text { O2. Possibility to clearly understand } \\
\text { reports A4.1.2. } \\
\text { O3. Output's statistical analysis A3.5., } \\
\text { A4.1., B4.1. }\end{array}$ \\
\hline & $\begin{array}{l}\text { B5. Tool hardware and } \\
\text { software } \\
\text { B6. Tool documentation } \\
\text { B7. User features }\end{array}$ & \\
\hline
\end{tabular}

Table 1: The proposed model of simulation software evaluation in relation to foundation models. 
What is common for both models is the division into three main categories of software evaluation criteria: modeling capabilities, simulation capabilities and output analysis. Within these categories, sub-category criteria are formed based on the original models or added as new items. The primary reason for opting for the three (same) categories (modeling and simulation capabilities, input/output analysis) was the fact that they describe the main features of explored tools relevant to the academic environment, in a comprehensive yet rather simple way adapted to the environment and the purpose of this research. Several subcategories were added, for example M10. Intuitiveness of objects. All subcategories that were not part of the original models were added to maximize the level of adaptation to a specific research purpose. Furthermore, there are subcategories in the research model that were created as a combination of the source model sub-categories, such as O3. Output statistical analysis (A3.5. Statistical facilities, A4.1. Input and output capabilities, B4.1. Output analysis).

The authors have estimated that there is no need to drill-down the subcategories in this research phase and that this type of grouping is beneficial to the research model. Finally, re-arrangement on model levels was done by adapting characteristics from the category level in the original model to fit a sub-category level in the newly created model; for example B6. Tool documentation was included in M13. Online help. Categories that were excluded from the models during the adaptation phase were perceived as less relevant in this phase of research, as they are not crucial for simulation tools' evaluation in academic surrounding or for assessing their suitability for academic purposes, i.e., A.1. Hardware and software considerations were excluded from the first model whereas B1. Tool capabilities, B5. Tool hardware and software, and B7. User features were excluded from the second.

\section{Selecting discrete event simulation tools for model testing}

The purpose of the developed research model is to provide the basis for simulation tools' evaluation in terms of inspecting effectiveness of explored tools and determining their suitability for academic purposes. Hence, the suggested model was tested on two widely used simulation tools, Arena ${ }^{\circledR}$ Simulation Software by Rockwell Automation and ExtendSim by Imagine That. Both were used for teaching discrete event simulation in the Business Process Simulations course and therefore it was crucial to explore whether the suggested model will provide a significant distinction between the two software tools.

Both tools support discrete event simulation giving the possibility to build models with entities competing for limited resources and are available to our institution free of charge, Arena licensed as Arena Student version (providing 
the functionality of Arena Professional Edition) and ExtendSim through ExtendSim Adopter licensing program (also a professional, "full" version).

\subsection{Arena ${ }^{\circledR}$ Simulation Software by Rockwell Automation (USA)}

Among other features, Arena enables business process simulations. It was designed to provide analysis and an insight into changes that could or should be done with regard to company's business processes, regardless of company's line of work. Typical examples of Arena usage include production system analysis as well as analysis of complex customer relationship systems or supply chains. The tool can also be used for performance estimates based on key indicator data, such as cost, cycle time, etc., as well as for identifying problems in business processes and planning and developing resources (as listed in Arena Standard Edition Users Guide by Rockwell Software [11]).

Potential users of the tool are consultants and analysts in production or business process managers, as well as industrial and system engineers. Vendor's official materials list Arena's features in business process design/modeling/simulation in the following manner:

1. process modeling - defining, documenting, communicating;

2. process simulation - gaining an insight into future system performance in order to understand complex connections between system components and to identify improvement possibilities;

3. process visualization - presenting activities including dynamic graphics;

4. process analysis - comparing an as-is system and its to-be version, in order to make grounded business decisions.

Arena's toolbar consists of "standard" elements for saving, opening previously saved models, zooming, object drawing, text editing, etc. (see Figure 1). What differentiates Arena from other simple modeling tools is its capability to start and manage the business process simulation model, as well as an option to create graphs for in-depth process analysis.

There are three lists inside the modeling objects toolbar:

1. Basic Process, Advanced Process and Advanced Transfer - contains shapes needed for process modeling (modules). A drag-and-drop technique is used to create diagrams.

2. Reports - contains reports that can be generated upon simulating the modeled process.

3. Navigate - enables different possibilities of presenting the created model. 
Two main modeling areas in Arena are a flowchart view and a spreadsheet view. A flowchart view will contain all graphical presentation modeling and simulating, while a spreadsheet view will collect and present model data: time, expense, etc.

\subsection{ExtendSim by Imagine That (USA)}

Very similar usage scenarios are possible in ExtendSim as well. Those include: anticipating workflow and business activity result, an insight into a particular problem or repercussions of a business activity, stimulating creative thinking, identifying problem areas before implementation of a new technology or methodology, evaluating ideas, optimizing activities, etc. [8]. ExtendSim's features include built sets of objects that enable fast model building, an adaptable graphic interface that describes model relationships, unlimited hierarchical decomposition, which makes models easier to understand and to model. Also, there are 2D and 3D animations, the possibility of setting parameters during simulation, the possibility of creating new objects and blocks, reporting, business process optimizing, etc. (as listed in ExtendSim 8 User Guide [7]). Furthermore, ExtendSim supports a discrete event as well as continuous simulation.

As for Arena, the basic possibilities and user interface for modeling, simulating, visualizing, and analyzing models are shown in Figure 2. ExtendSim's layout is dominated by the modeling area. The toolbar also contains "standard" elements for saving, opening previously saved models, zooming, etc. One is also allowed to start, speed or stop simulation, in the toolbar. It is possible to open object libraries, one by one or all at the same time, by clicking on Library. Likewise, diagrams are constructed using the dragand-drop technique.

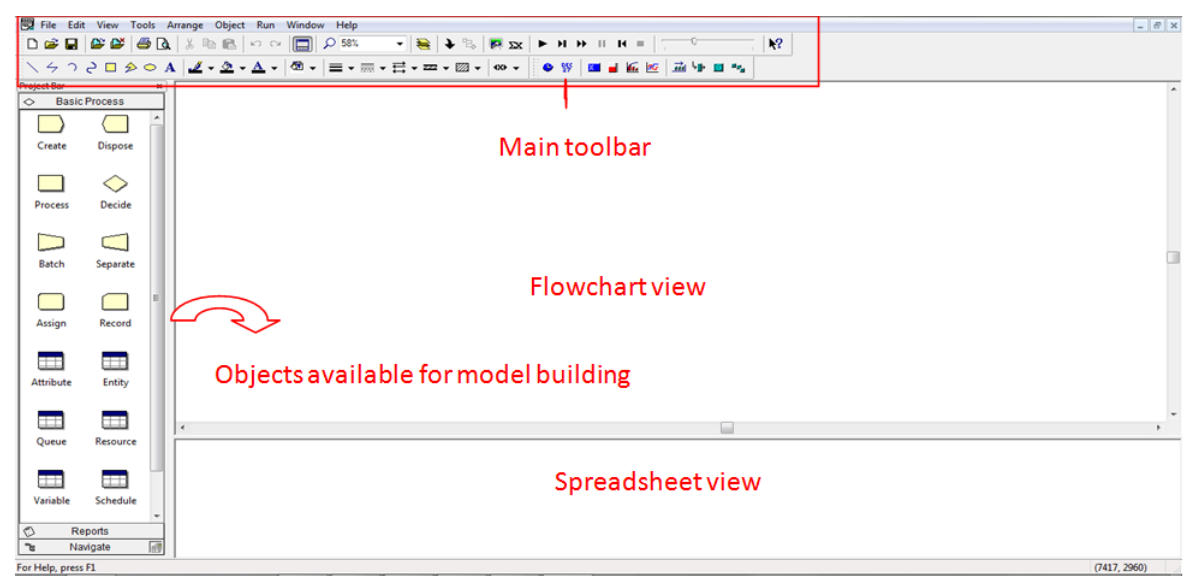

Figure 1: Arena user interface. 


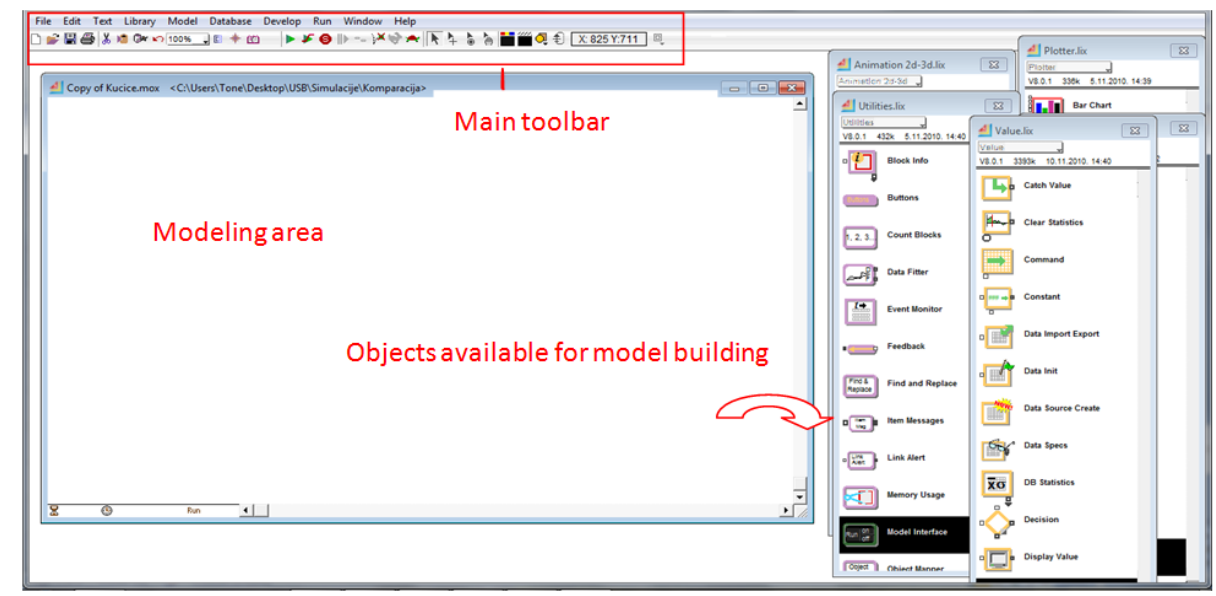

Figure 2: ExtendSim user interface.

\section{Research methodology}

Even though steps in model building and simulating are practically the same, regardless of the chosen tool, each one does not suit every business situation [9] - or in this case - the academic purpose. As already stated, the goal of this research was to evaluate appropriateness of the tools in an academic surrounding by measuring and analysing subjective and objective indicators, and to conclude which one suits this purpose better. Even if students do prefer one tool over the other it does not mean the tool they prefer is better in terms of learning outcomes and effectiveness.

\subsection{Research procedure}

Arena and ExtendSim were used for building the same model and then evaluated by first year Masters' students in Information Management at the Faculty of Economics, University of Split enrolled in the Business Process Simulation course. Out of 28 students enrolled in the course in 2013/2014, 24 participated in the study -16 male and 8 female students. It took about 15 minutes to build simulation models although the tasks were not time-limited. The length of the online survey, as estimated by the online surveying tool SurveyGizmo, was 6 minutes per tool, i.e., 12 minutes altogether.

Research was conducted in a controled environment, during one of course classes in a computer lab. During previous class, i.e., one week earlier students were instructed that research will be conducted. They were also asked to recall 
how to use both tools. During research exercise, Mousotron tool [1] was used in order to collect objective indicators of usage. Mousotron enables tracking of different activities performed using a computer mouse, such as the total number of left, right and double clicks, speed achieved, etc.

The challenge was to choose a process that is simple enough to evaluate the tools' basic capabilities. For this purpose, a simple tollbooth ("textbook") model was adapted, i.e., the process of a number of cars passing through tollbooths was modeled and simulated. Although elementary, it covers all the features, i.e., modeling, simulation and output analysis in order to evaluate selected tools. Students were randomly assigned to two groups and were given detailed, stepby-step instructions or the scenario for building the model using both tools first using ExtendSim, then Arena or vice versa.

The simulation model in Arena is presented in Figure 3. The simulation is performed once, with the duration of 12 hours. Arrival of cars is presented using the object Create, which is a starting object when modeling a business process in Arena. Arrival is defined by the exponential function, with one car arriving every three minutes. The maximum number of arrivals is 200. By setting up the object Entity, cars are brought into the model. The probability of selecting a tollbooth is modeled using the object Decide. In this case, each car has an option of selecting one of tollbooths. There is a $50 \%$ chance that a car will select the first tollbooth, and the same percentage that it will select the second one. After selecting one of tollbooths, the car goes through the process. This is presented using the object Process. This activity's type is Seize Delay Release, which means that once a car has selected a tollbooth and come in the process, it fetches a resource called "Worker", which was previously added to the model. The worker at a tollbooth performs an action with a certain delay and is released upon performing the activity. A delay of 10 minutes is constant. The process is completed using the object Dispose, and cars are leaving tollbooths. A Plot is added to monitor both changes and activity flow. The simulation is run and upon its completion a set of questions is posed for students to answer: what is the average and the maximum waiting period; and how many cars went through the model during the set period of 12 hours?

The simulation model in ExtendSim is presented in Figure 4. The simulation is performed once, with the duration of 12 hours. Before process modeling, the object Execute is added to the model. This object is prerequisite and enables performing the simulation. Arrival of cars is modeled using the object Create, which is a starting object when modeling and simulating in ExtendSim. Arrivals are defined by the exponential function, with one car arriving every three minutes. The maximum number of arrivals is 200. Upon 
arrival of cars, a queue is made; therefore, one is added to the model, using the object Queue. This queue is related to a resource that is engaged to perform a business activity. This item is modeled using the object Resource Pool where the number of Workers is set to two (one worker for one tollbooth). After this, the queue is connected to the modeled resource. After placing the queue in the model, the object Select Item Out is chosen and added. By using this object, it is indicated that a car is redirected to one of the two tollbooths available, with the same probability of choosing one or another tollbooth. After choosing a tollbooth, cars go through the activity of passing it, which is displayed using the object Activity. The maximum number of objects going into the activity is 1. A delay of 10 minutes is constant. ExtendSim requires adding an object for releasing an engaged resource upon completing the activity. For this, object Resource Pool Release is used. At the end, the object Exit was added, which denotes that a car has left the process. Also, a diagram was added and connected to the object Queue, in order to get a deeper insight into performed activities and the simulation itself. The simulation is run and upon its completion the same set of questions is asked as for Arena.

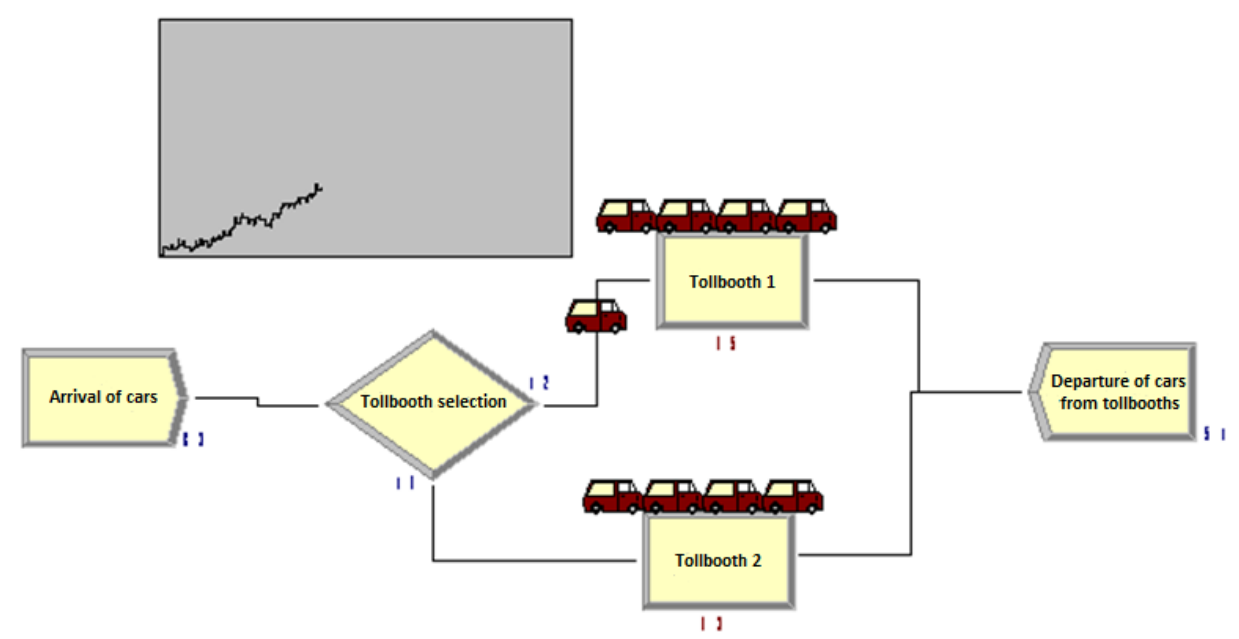

Figure 3: Simulation model in Arena. 


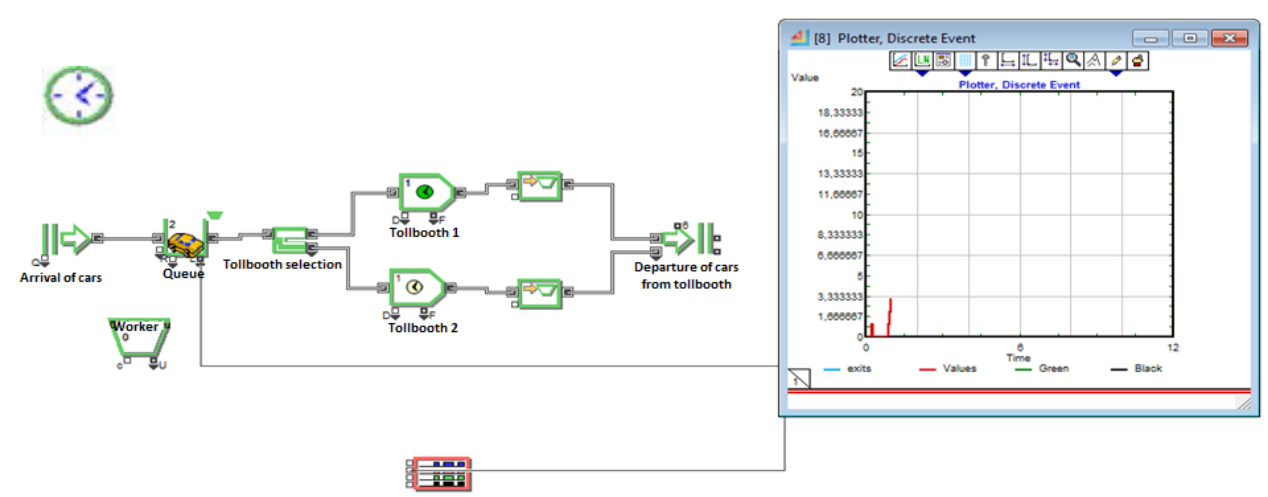

Figure 4: Simulation Model in ExtendSim.

Upon finishing business process modeling and simulation in Arena and ExtendSim (or vice versa), students have taken a survey where they were required to attach a print screen of recorded Mousotron statistics. The online survey was created to test the efficiency of the tools using the proposed model. A theoretical basis of the model is presented in Section 2.

The survey consisted of nine questions divided into four categories: (1) General information, (2) Modeling capabilities, (3) Simulation capabilities, and (4) Capabilities of output analysis. General information questions deal mostly with students' subjective estimation of their own theoretical and practical knowledge of business process simulations, while the remaining parts of the survey questioned one's ability and preference to model, simulate and analyze output using a particular tool. The modeling capabilities (modeling characteristics, problems and tasks) of the two tools were evaluated using two different sets of questions. Students estimate how good Arena/ExtendSim is with regard to the modeling process (questions M1 to M7 on a five-point performance scale from 1 to 5, i.e., very hard, hard, not easy-not hard, easy, very easy) and modeling support (questions M8 to M13 on a qualitative scale from 1 to 5, i.e., bad, satisfying, good, very good, excellent). With regard to simulation and output analysis features, the same scale was used as for questions M8 to M13 (bad, satisfying, good, very good, and excellent).

\subsection{Research results}

With regard to students' subjective estimation of their own theoretical knowledge of discrete event simulation students assess that they mostly spent from 8 to 16 hours (42\%) and up to 8 hours (29\%), while $12 \%$ of them spent 16 to 20 hours, $13 \% 20$ to 24 hours and $4 \%$ more than 24 hours learning the theoretical foundations of discrete event simulations. Subjective estimation of the time spent using business process simulation tools was done for both tools, 
including the time spent doing exercises in class and individually at home. For ExtendSim, the time students spent is slightly higher, namely: up to 4 hours (29\%), 4 to 8 hours (50\%), 8 to 12 hours (13\%), and more than 12 hours (8\%). In Arena, students spent up to 4 hours (71\%), 4 to 8 hours (25\%), 8 to 12 hours (4\%). Students estimated their ability to use Arena and ExtendSim on a scale from 1 to 5 (i.e., very weak to excellent) per category of features. Results are presented in Table 2. In general, ExtendSim is rated better in every aspect, meaning that students perceive they know how to use the tool better than Arena. This is probably because they spent more time using it and therefore consider themselves more competent in using ExtendSim. Regardless of the tool, students assess their ability to model slightly better than abilities to simulate and analyse output, respectively.

\begin{tabular}{|l|c|c|c|c|}
\cline { 2 - 5 } \multicolumn{1}{c|}{} & \multicolumn{2}{c|}{ Arena } & \multicolumn{2}{c|}{ ExtendSim } \\
\cline { 2 - 5 } \multicolumn{1}{c|}{} & Mean & SD & Mean & SD \\
\hline Modeling & 2.04 & 0.859 & 3.13 & 0.900 \\
\hline Simulating & 2.00 & 0.978 & 2.67 & 0.816 \\
\hline Output analysis & 1.83 & 1.007 & 2.50 & 0.933 \\
\hline
\end{tabular}

Table 2: Estimated ability to use Arena and ExtendSim per category.

Categories of tools' capabilities and characteristics evaluated in research are elaborated. Results of tools' comparison per category are presented in Table 3. Modeling allows representation of different forms of behavior within the model. This refers to features such as procedures of building models, generating transactions, branching, merging and separating process flow, assigning resources to activities, changing their properties in time, importing data, and defining different transaction flows through the model depending on their characteristics. Students who have used both tools to build a very simple model described earlier in the paper, perceive ExtendSim better across all aspects of the modeling process and modeling support provided by the tool. Building a simulation model is inherently an iterative process, so the students themselves have built a model gradually according to the prepared script. This approach made it possible for students to launch the simulation model in the early stages of model construction, while the animation features and dynamic presentation of data in the model facilitated the identification and correction of errors (i.e., model verification) in the process of simulating a given scenario. The purpose of a very simple analysis of data in terms of replies to a couple of simple questions was to assess the efficiency and effectiveness of the simulated scenario, and to point to the importance of a built-in functionality of the tool for automatic collection of simulated process data and their statistical analysis. Again, upon using both tools for the simulation and output analysis students evaluated ExtendSim to be better across all features. 
Figure 5 illustrates assessments per feature for both tools. Overall, ExtendSim was graded better in all subjective indicators, i.e., students perceive ExtendSim as a better tool in general and per feature. This result could be explained by several factors. First of all, ExtendSim was planned by the curriculum and studied later in the semester than Arena, and therefore closer to the research dates. It is possible that students simply recall ExtendSim better and therefore perceive it better due to that fact. Secondly, during the course Simulation of Business Processes, ExtendSim was studied through individual (home) work. Hence, it is possible that students have spent much more time performing tasks in ExtendSim and are therefore more familiar with the tool. Of course, it is possible that the above mentioned factors are not correlated with subjective opinion of surveyed students and that ExtendSim is simply more attractive (user interface) and superior (in terms of functionalities) in relation to Arena, regardless of the frequency and the time spent using the tool.

\begin{tabular}{|c|c|c|c|c|c|}
\hline & & \multicolumn{2}{|c|}{ Arena } & \multicolumn{2}{|c|}{ ExtendSim } \\
\hline & & Mean & SD & Mean & $\mathrm{SD}$ \\
\hline \multirow{7}{*}{$\begin{array}{l}\text { Modeling } \\
\text { process }\end{array}$} & M1. Using the tool & 2.21 & 0.779 & 3.38 & 0.824 \\
\hline & M2. Procedure of model building & 2.42 & 1.100 & 3.46 & 0.884 \\
\hline & M3. Studying how to use the tool & 2.29 & 1.083 & 3.46 & 0.977 \\
\hline & M4. Model verification & 2.13 & 0.900 & 2.88 & 0.741 \\
\hline & M5. Importing resources into a model & 2.46 & 1.103 & 3.37 & 1.013 \\
\hline & M6. Importing data into a model & 2.38 & 0.924 & 3.33 & 1.007 \\
\hline & M7. Adding and editing model data & 2.21 & 0.977 & 3.42 & 1.018 \\
\hline \multirow{6}{*}{$\begin{array}{l}\text { Modeling } \\
\text { support }\end{array}$} & M8. Number and types of templates & 1.92 & 0.717 & 3.04 & 0.908 \\
\hline & M9. Level of details during modeling & 2.08 & 0.929 & 3.37 & 1.135 \\
\hline & M10. Intuitiveness of objects & 2.42 & 0.974 & 3.25 & 0.944 \\
\hline & $\begin{array}{l}\text { M11. Automatic tracking of basic model } \\
\text { performance }\end{array}$ & 2.21 & 0.932 & 3.08 & 0.776 \\
\hline & $\begin{array}{l}\text { M12. Automatic tracking of a larger number of } \\
\text { experiments }\end{array}$ & 2.17 & 0.761 & 3.00 & 0.834 \\
\hline & M13. On-line help & 2.00 & 0.933 & 2.58 & 0.974 \\
\hline \multirow[t]{8}{*}{ Simulation } & $\begin{array}{l}\text { S1. Performance display (resource/time usage, } \\
\text { quality) }\end{array}$ & 2.38 & 0.770 & 3.13 & 0.680 \\
\hline & $\begin{array}{l}\text { S2. Importing and choosing probability } \\
\text { distribution }\end{array}$ & 2.21 & 0.779 & 2.96 & 0.999 \\
\hline & S3. Model animation & 2.17 & 1.007 & 3.33 & 0.816 \\
\hline & S4. Adaptability to model changes & 2.42 & 0.881 & 3.25 & 0.847 \\
\hline & S5. Reliability (animation) & 2.17 & 0.816 & 3.17 & 0.917 \\
\hline & S6. Speed of executing the simulation & 2.54 & 0.833 & 3.75 & 0.944 \\
\hline & S7. Dynamic objects of the model & 2.33 & 0.761 & 3.13 & 0.797 \\
\hline & S8. Dynamic display of statistical data & 2.08 & 0.717 & 3.50 & 0.780 \\
\hline \multirow{3}{*}{$\begin{array}{l}\text { Output } \\
\text { analysis }\end{array}$} & O1. Report's format & 1.87 & 0.741 & 3.00 & 0.834 \\
\hline & O2. Possibility to clearly understand reports & 2.00 & 1.063 & 2.96 & 0.908 \\
\hline & O3. Output's statistical analysis & 1.96 & 0.999 & 2.79 & 0.932 \\
\hline
\end{tabular}

Table 3: Estimated features of Arena and ExtendSim. 
On the other hand, Arena is far superior to ExtendSim when it comes to objective indicators (figure 6): faster model building, less clicks and less movement are sound indicators. It took 3.08 minutes less time to go through the script and build a model in Arena than in ExtendSim which is $18.67 \%$ faster. In Arena almost $90 \%$ less mouse scrolling and $21 \%$ fewer left mouse button clicks was necessary to build the simulation model.

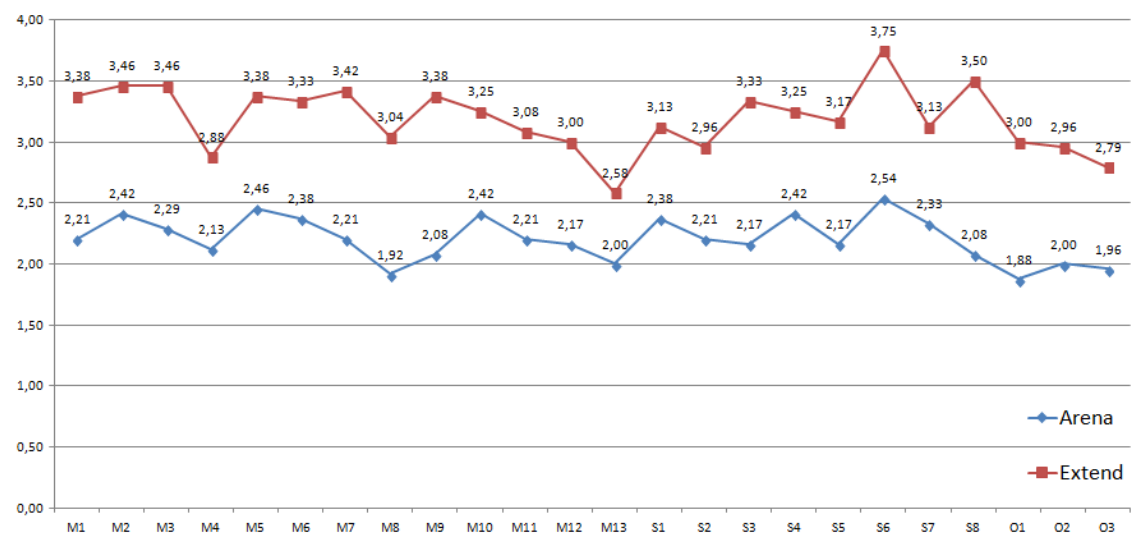

Figure 5: Comparison of feature assessments for Arena and ExtendSim.

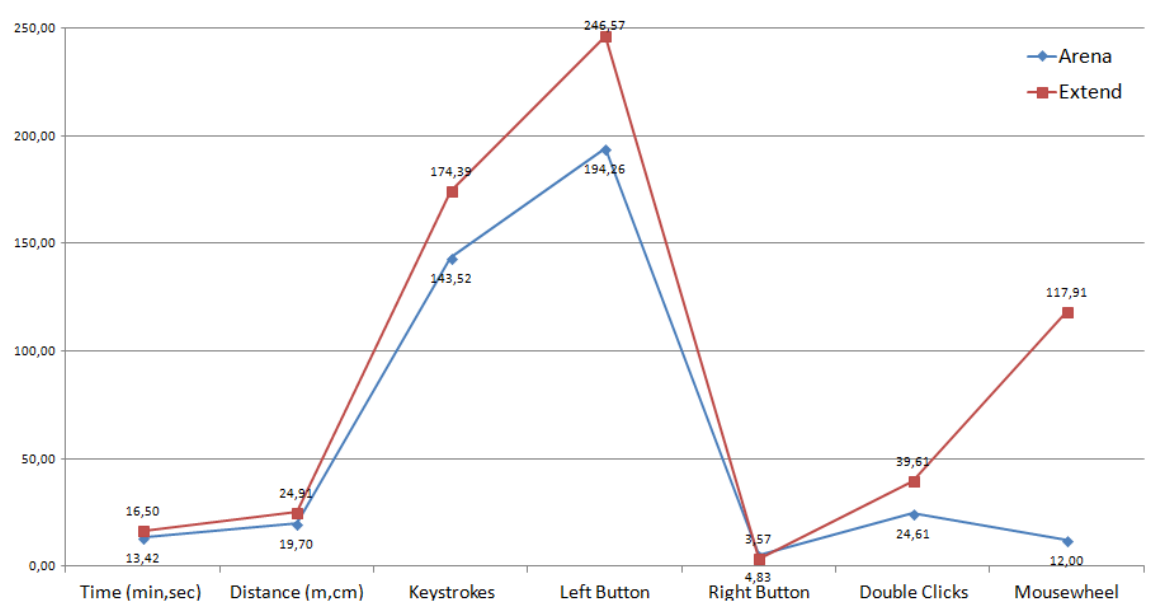

Figure 6: Comparison of objective indicators for Arena and ExtendSim.

Research experiments have confirmed that the suggested model is efficient in a subjective comparison of simulation tools. The model was tested using a simple, yet thorough end-to-end feature comparison provided by the simulation model. Tools were assessed and compared based on a defined set of criteria, which have been confirmed as a valuable combination of important tools' 
characteristics. This in particular is the strength of the suggested model. A clear categorization of important characteristics is provided, with the model being adapted for a specific purpose, i.e., comparing simulation tools in an academic environment.

By using the proposed evaluation model a clear, significant difference was found between inspected tools in terms of subjective criteria. Considering the difference in subjective evaluations brought forth by the research, we note the importance of objective evaluation as well. In this particular exercise, although student attitudes point that one tool is preferred to other, for teaching effectiveness it is important to take into account objective results as well.

\section{Conclusion}

Computer simulation enables us to gain a valuable insight into business processes, possible problems that might occur and identify potential for improvements. Furthermore, it is an important tool for decision-making since it can provide insights into "to-be" systems, without interfering with the process itself. This feature allows decision-makers to make decisions based on concrete data and not on intuition. One of the most important advantages of simulating business processes in this way is that the entire process of analysis can be done in steps. It is possible to start with a simple approximation of a business process, and upgrade the model along with user's growing understanding of the modeled process. By detailing out a model, it becomes very similar to the realworld process it represents. It is possible to effectively teach and study discrete event simulation by using tools such as Arena and ExtendSim. This research has compared these two tools when they are used in academic surrounding and for modeling an extremely simple process appropriate for this type of usage. A new research model of simulation software evaluation is suggested, developed based on previous research related to BPS tools and authors' experience in regard to the field of research. This model consists of three categories: modeling and simulation capabilities of the explored tools, and tools' input/output analysis possibilities, all with respective sub-criteria. It gives a new dimension to comparing simulation tools in academic surrounding and is therefore only the beginning of a much broader exploration of the usage and acceptance as well as the role of simulations in teaching business students.

Although partially derived from models used for evaluating tools in corporate surrounding, the created model is primarily predetermined for an academic environment. The model was created with the aim to inspect tools' effectiveness and to help determine which one is more suitable for students, meaning that questions are contextualized in a way that they address only 
particular characteristics related to academic use. Some categories important for evaluating BPS tools were not explored by this model, but could be very important for business environment (e.g., financial features, software compatibility). Finally, the model is adapted for a simple business process, due to its primary purpose. In short, results have shown that ExtendSim is superior to Arena when it comes to subjective indicators, while a vice-versa relation works when it comes to objective indicators. This means that, even though students have spent less time and done less movements for building the model in Arena, they still prefer ExtendSim and perceive it as a better tool. Whether these results are related to a fresher memory of ExtendSim among students or to the length of time spent in individual usage of the tools, still remains open.

We plan to advance the research by repeating and verifying the findings and investigating correlations between the variables. For example, a valid question is whether there is a connection between the results achieved on the theoretical test with subjective and objective indicators. Another relevant question is if the results would differ significantly if a more demanding scenario/process was evaluated, i.e., if it increases or decreases the difference of the compared subjective and objective indicators.

\section{References}

[1] Blacksun software official website, 2014. [online] Available at: http://www.blacksunsoftware.com/mousotron.html [Accessed on 2 September 2014].

[2] Bosilj-Vukšić, V., Čerić, V. \& Hlupić, V., 2007. Criteria for the evaluation of business process simulation tools. Interdisciplinary Journal of Information, Knowledge, and Management, 2, 73-88.

[3] Bradley, P., Browne, J., Jackson, S. \& Jagdev, H., 1995. Business process reengineering - A study of the software tools currently available. Computers in Industry, 25(3), 309-30.

[4] Gupta, A., Singh, K. \& Verma, R., 2010. A critical study and comparison of manufacturing simulation softwares using analytic hierarchy process. Journal of Engineering Science and Technology, 5(1), 108-129.

[5] Hlupić, V., 1999. Simulation software: User's requirements. Computers \& Industrial Engineering, 37, 185-88.

[6] Hlupić, V., Paul, R.J. \& Irani, Z. (1999). Evaluation framework for simulation software. International Journal of Advanced Manufacturing Technology, 15, 366382.

[7] Imagine That Inc., 2010. ExtendSim 8 User Guide. Imagine That Inc.

[8] Imagine That Inc., 2012. Products: ExtendSim overview. [online] Available at: http://wWw.extendsim.com/prods_overview.html [Accessed on 16 August 2014]. 
[9] Jansen-Vullers, M.H. \& Netjes, M., 2006. Business process simulation - A tool survey. [online] Available at: http://www.researchgate.net/profile/Monique Jansen-

Vullers/publication/228358579_Business_process_simulationa_tool_survey/lin ks/004635190a2a13e2af000000 [Accessed on 19 August 2014].

[10] Pidd, M., 1989. Choosing discrete simulation software. OR Insight, 2(3), 22-23.

[11] Rockwell Software, 2002. Arena Standard Edition Users Guide. Rockwell Software.

[12] Verma, R. \& Gupta, A.S.K., 2008. Simulation software evaluation and selection: A comprehensive framework. J. Automation \& Systems Engineering, (2-4), 221-34. 